\title{
Estoque de carbono orgânico e atributos físicos do solo em sistema de integração lavoura-floresta com eucalipto no Cerrado Mineiro
}

\author{
Organic carbon stock and physical soil attributes in a crop-forest and eucalyptus integration
} system in the Cerrado Mineiro

Reserva de carbono orgánico y atributos físicos del suelo en un sistema de integración cultivobosque y eucalipto en el Cerrado Mineiro

\begin{abstract}
Resumo
O sistema de integração lavoura-floresta (ILF) integra o componente florestal e agrícola. Este estudo objetivou avaliar os estoques de carbono orgânico e atributos físicos do solo sob sistema de integração lavoura-floresta, no sudoeste de Minas Gerais. O estudo foi desenvolvido na Fazenda Experimental da Universidade do Estado de Minas Gerais, unidade Acadêmica de Passos. O delineamento experimental utilizado foi em blocos ao acaso, com três tratamentos e sete repetições. O efeito desse sistema sobre os atributos do solo foi testado por meio de análise de variância. Avaliaram-se os efeitos dos tratamentos considerando a posição de linha e entrelinha. Quando houve efeito significativo $(\mathrm{P}<0,05)$ de tratamento, procedeu-se teste de Tukey-Kramer a $\mathrm{P}<0,05$ e entre as posições teste $\mathrm{t}$ a $\mathrm{P}<0,05$, utilizando-se o Os atributos físicos alterados pelo sistema ILP foram densidade do solo, volume total do solo e temperatura. A mata primaria apresentou teores e estoque de COS superior aos demais tratamentos. O estoque total de COS não apresentou diferença entre o sistema ILP e a mata primária, sinalizando a sustentabilidade deste sistema de produção.
\end{abstract}

Palavras-chave: Sustentabilidade; Mudanças climáticas; Matéria orgânica.

\begin{abstract}
The crop-forest integration system (ILF) integrates the forestry and agricultural component. This study aimed to evaluate organic carbon stocks and soil physical attributes under a crop-forest integration system, in southwestern Minas Gerais. The study was carried out at the Experimental Farm of the Universidade do Estado de Minas Gerais, Academic Unit of Passos. The experimental design used was in randomized blocks, with three treatments and seven replications. The effect of this system on soil attributes was tested by means of analysis of variance. The effects of treatments were evaluated considering the position of row and between rows. When there was a significant effect $(\mathrm{P}<0.05)$ of treatment, the Tukey-Kramer test was performed at $\mathrm{P}<0.05$ and between the test positions $\mathrm{t}$ and $\mathrm{P}<0.05$, using the Physical attributes altered by the system ILP were soil density, total soil volume and temperature. The primary forest showed higher COS contents and stock than the other treatments. The total stock of COS showed no difference between the ILP system and the primary forest, indicating the sustainability of this production system.
\end{abstract}

Keywords: Sustainability; Climatic changes; Organic material.

\section{Resumen}

Incluir o resumo em espanhol. El sistema de integración cultivo-bosque (ILF) integra el componente forestal y agrícola. Este estudio tuvo como objetivo evaluar las reservas de carbono orgánico y los atributos físicos del suelo 
bajo un sistema de integración cultivo-bosque, en el suroeste de Minas Gerais. El estudio se llevó a cabo en la Granja Experimental de la Universidade do Estado de Minas Gerais, Unidad Académica de Passos. El diseño experimental utilizado fue en bloques al azar, con tres tratamientos y siete repeticiones. El efecto de este sistema sobre los atributos del suelo se probó mediante análisis de varianza. Los efectos de los tratamientos se evaluaron considerando la posición de hilera y entre hileras. Cuando hubo un efecto significativo $(\mathrm{P}<0.05)$ del tratamiento, se realizó la prueba de Tukey-Kramer en $\mathrm{P}<0.05$ y entre las posiciones de prueba t y $\mathrm{P}<0.05$, utilizando los atributos físicos alterados por el sistema ILP fueron densidad del suelo, total volumen y temperatura del suelo. El bosque primario mostró mayores contenidos de COS y existencias que los otros tratamientos. El stock total de COS no mostró diferencia entre el sistema ILP y el bosque primario, lo que indica la sostenibilidad de este sistema de producción.

Palabras clave: Sustentabilidad; Cambios climáticos; Materia orgánica.

\section{Introdução}

O sistema de integração lavoura-floresta (ILF) ou silviagrícola integra os componentes florestal e agrícola pela consorciação de espécies arbóreas com cultivos agrícolas anuais ou perenes (Balbino et al., 2011). Estes são como uma ferramenta que promove a recuperação dos solos os quais reduz a erosão do solo e uma alternativa de renda para agricultores (Weimann et al., 2017). Devido aos benefícios socioambientais e econômicos os sistemas de cultivos com arbóreas perenes é uma estratégia para solucionar problemas como a mudanças climáticas globais, degradação do solo, poluição das águas por fertilizantes, redução da erosão, desertificação, diminuição da biodiversidade e insegurança alimentar (Lal et al., 2007).

O COS (carbono orgânico do solo) corresponde a cerca de 58\% da matéria orgânica do solo, sendo composta por plantas, micro-organismos e animais, em diferentes fases de decomposição, desde resíduos frescos até altamente modificados (FAO, 2017). O COS é um relevante indicador da qualidade do solo, uma vez que dá suporte para as funções essenciais do solo, como estabilização da estrutura do solo, proteção dos microagregados (Bossuyt et al., 2005), densidade do solo, liberação de nutrientes, infiltração e armazenamento de água (Chevallier et al., 2004). Desta maneira, o COS é fundamental para garantir a saúde do solo, como a diversidade e fertilidade e para manter a produção de alimentos.

A mudança no uso da terra geram a dimunição (50-61\%) do carbono orgânico do solo (Hombegowda et al., 2016). Além do efeito degradador na qualidade do solo que conduz a perda contínua de matéria orgânica o que é uma relevante preocupação devido às emissões de gases de efeito estufa (Lal, 2004). A mudança no sistema de manejo do solo pode ser uma ferramenta para reduzir o estoque de carbono orgânico do solo (Assunção et al., 2019; Troian et al., 2020; Ferreira, 2020).

O melhor gerenciamento do solo na agricultura ocorre com respiração reduzida (Ballantyne et al., 2017), menores emissões de uso da terra, como na florestação (Piao et al., 2018). Esses são itens desejaveis na identificação de sistemas agrícolas capazes de estocar carbono (C) em associação com a estabilidade de C (Balbino et al., 2012), o que justifica a realização deste estudo. Portanto, o objetivo deste estudo foi avaliar os atributos físicos e teores e estoque de carbono orgânico do solo (COS) em área de integração lavoura-floresta com eucalipto, na cidade de Passos, sudoeste de Minas Gerais, Brasil.

\section{Metodologia}

A área de estudo está localizada na Fazenda Experimental da Universidade do Estado de Minas Gerais, Unidade de Passos, nas coordenadas geográficas $20^{\circ} 44^{\prime} 25^{\prime \prime} \mathrm{S}$ de latitude e $46^{\circ} 37^{\prime} 44^{\prime \prime}$ longitude e com altitude 875 metros. O clima da região segundo o sistema de Köppen (1948) é tipo Cwa, ou seja, clima subtropical/tropical de altitude com inverno seco e verão quente (Alvares et al., 2011). A Precipitação pluviométrica segundo INMET (Instituto Nacional de Meterologia, 2019) anual está entre 1.100 a $1.700 \mathrm{~mm}$ e a temperatura anual média mínima é de $13,4^{\circ} \mathrm{C}$ e temperatura média máxima é de $25,2^{\circ} \mathrm{C}$. O Solo foi classificado como Latossolo Vermelho Amarelo distrófico e com declividade média de 6\%. (Empresa Brasileira de Pesquisa Agropecuária-EMBRAPA, 2006). A caracterização química do solo amostrado é apresentada Tabela 1. O sistema ILF foi implantado em 2011. A adubação de plantio consistiu em $50 \mathrm{~g}$ de superfosfato simples por cova e a adubação de cobertura de $3 \mathrm{~g}$ de boro (B) por planta em 2 anos seguidos. 
Tabela 1. Atributos de fertilidade do solo da área em estudo.

\begin{tabular}{|c|c|c|c|}
\hline & $\mathrm{pH}^{1}$ & $\mathbf{P}$ & CTC \\
\hline Mata & 4,55 & 6,33 & 59,86 \\
\hline Bosque & 4,88 & 25,86 & 52,71 \\
\hline \multirow{2}{*}{ Eucalipto } & 4,22 & 8,86 & 55,00 \\
\hline & $\mathbf{A l}^{3}$ & $\mathrm{H}+\mathrm{Al}^{4}$ & V \\
\hline Mata & 4,33 & 33,33 & 44,33 \\
\hline Bosque & 2,86 & 29,29 & 44,71 \\
\hline \multirow[t]{2}{*}{ Eucalipto } & 5,29 & 41,71 & 27,29 \\
\hline & $K^{2}$ & $\mathrm{Ca}^{2}$ & $\mathbf{S}$ \\
\hline Mata & 2,4 & 16,67 & 3,83 \\
\hline Bosque & 2,87 & 21,57 & 4,00 \\
\hline \multirow[t]{2}{*}{ Eucalipto } & 1,87 & 9,43 & 4,86 \\
\hline & $\mathrm{Mg}^{2}$ & S.B & \\
\hline Mata & 7,83 & 26,43 & \\
\hline Bosque & 4,57 & 23,43 & \\
\hline Eucalipto & 3,57 & 18,14 & \\
\hline
\end{tabular}

${ }^{(1)} \mathrm{pH}$ em $\mathrm{CaCl}_{2} 0.01 \mathrm{~mol} \mathrm{~L}^{-1}$ em uma taxa de $1: 2.5(\mathrm{~m} / \mathrm{v}) ;{ }^{(2)} \mathrm{P}, \mathrm{K}^{+}, \mathrm{Ca}^{2+}$ e $\mathrm{Mg}^{2+}$ extraído por resina trocavél; ${ }^{(3)} \mathrm{Al}^{3+}$ extraído por $\mathrm{KCl}$ $1 \mathrm{molL}^{-1} ;{ }^{(4)} \mathrm{H}+\mathrm{Al}$ extraído pelo método $\mathrm{SMP} ;{ }^{(5)} \mathrm{SB}=$ soma dos cátions básicos; ${ }^{(5)} \mathrm{CTC}=$ capacidade de troca de cátions; ${ }^{(5)} \mathrm{V}=$ saturação de bases, são indicadores de fertilidade do solo. Fonte: Autores.

A área experimental é composta por cinco bosques de eucaliptos (Eucalyptus urophylla), com $50 \mathrm{~m}$ de comprimento e cada um deles formados por três linhas de eucalipto no espaçamento de 2,0 m x 2,0m com $24 \mathrm{~m}$ de distância de um bosque para o outro, conforme apresentado na Figura 1. Os bosques são destinados as culturas anuais e na ausência desta encontra-se em pousio.

Figura 1. Croqui da área de integração Lavoura-Floresta

) Árvore (a) e vista geral da área experimental (b).
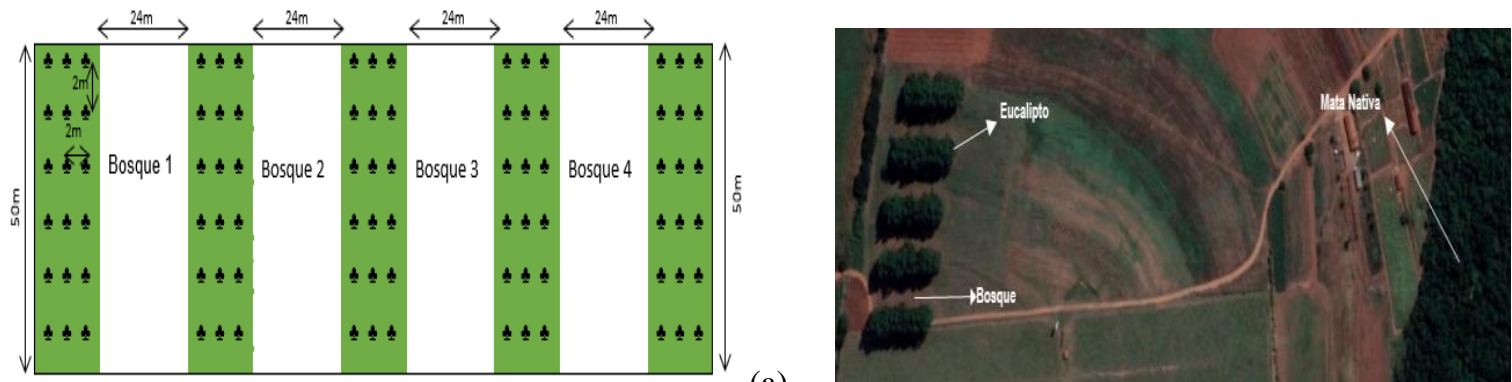

(b)

Fonte: Autores.

\subsection{Delineamento experimental}

O delineamento experimental foi em blocos ao acaso constituído por três tratamentos sendo linha de eucalipto, bosque e mata nativa, com sete repetições. Os bosques apresentam uma área a qual é destinada a culturas anuais, com título de comparação foi selecionada uma área de mata nativa, considerando-a como referência de massa original (Figura 1).

As amostragens do solo foram realizadas na linha de plantio dos eucaliptos, nos bosques (entrelinhas) e na mata nativa. Na posição linha de eucalipto e bosque as amostragens foram em pontos ao acaso e na mata nativa foram pontos ao acaso em ziguezague. As amostras indeformadas de solo foram coletadas em trincheiras com auxílio do anel de aço Kopecky (capacidade volumétrica de $84,4 \mathrm{~cm}^{3}$ ) e utilizadas para determinar a densidade do solo. As amostras deformadas foram 
coletadas na profundidade 0 a $20 \mathrm{~cm}$, com auxílio de uma espátula, e em seguida colocadas em saco plástico devidamente identificado, para as análises físicas dos solos.

\subsection{Parâmetros avaliados}

A granulometria do solo foi obtida pelo método da pipeta (Camargo et al., 1986). A densidade do solo (Ds) determinada pelo método do anel volumétrico. A Ds foi obtida pela razão entre a massa da amostra indeformada seca a $105^{\circ} \mathrm{C}$ e o volume cilíndrico (soma dos volumes ocupados pelos poros e partículas) de acordo a equação $\mathrm{Ds}=\mathrm{m} / \mathrm{V}$ onde que: Ds= densidade do solo, $\mathrm{g} \mathrm{cm}^{-3} ; \mathrm{m}=$ massa de solo seca; $\mathrm{V}=$ volume da amostra. Para retirada da amostra será usado o anel de kopeck e amostrador castelinho (Blake et al., 1986). A densidade de partícula (Dp) foi obtida pelo método do balão volumétrico (Blake \& Hartge, 1986). A Dp foi determinada por meio da relação entre a massa de uma amostra do solo seco (ms) e o volume ocupado pela fração sólida da amostra (Vs), segundo a equação $\mathrm{Dp}=\mathrm{ms} / \mathrm{Vs}$. A porosidade total do solo (VTP) foi obtida pela equação: VTP= 100(1-Ds/Dp), em que: Ds: densidade do solo e Dp: densidade de partícula (Vomicil, 1965). A temperatura do solo foi obtida com geotermômetro digital (TE07), tipo espeto com haste de penetração em aço inoxidável.

Os teores de carbono orgânico total do solo foram obtido a partir da matéria orgânica do solo (método do colorimétrico Camargo et al., 1986) considerando o valor de $58 \%$ de carbono. O estoque de carbono orgânico foi calculado por meio da seguinte expressão COS = C x (Ds) x v; onde: COS: carbono orgânico do solo $\left(\mathrm{Mg} \mathrm{ha}^{-1}\right)$; : teor de carbono do solo $\left(\mathrm{Mg} \mathrm{Mg}^{-}\right.$ ${ }^{1}$ ); Ds: densidade do solo $\left(\mathrm{Mg} \mathrm{m}^{-3}\right.$ ); volume considerado do solo: $\mathrm{m}^{3} \mathrm{ha}^{-1}$ (calculado a partir da profundidade da camada do solo (m) x $10.000 \mathrm{~m}^{2}$ há ${ }^{-1}$. Para compensar o efeito da compactação do solo, causada pelos tratos culturais e manejo dos estoques foram calculados pelo método da massa equivalente (Bayer et al., 2000), foi usado como referência as massas de solo de camadas correspondentes de uma área sob mata nativa.

\subsection{Análises estatísticas}

$\mathrm{O}$ delineamento experimental utilizado foi em blocos ao acaso, com três tratamentos e sete repetições. Em seguida foram submetidos ao teste de $\mathrm{F}$ ao nível de $(\mathrm{P}<0,05)$ de significância, quando houve um resultado significativo $(\mathrm{P}<0,05)$ de tratamento foi feito o teste de Tukey-Kramer $(\mathrm{P}<0,05)$ usando o pacote estatístico Sisvar (Ferreira et al., 2011).

\section{Resultados e Discussão}

\subsection{Efeitos do sistema ILF nos atributos físicos do solo}

A tabela 3 apresenta os resultados de análise de variância dos atributos físicos do solo para os tratamentos. Observouse, que ocorreu diferença estatística para densidade do solo, volume total de poros e temperatura. A ausência de significância entre as frações granulométricas demonstra que todos os tratamentos apresentam o mesmo potencial para sequestrar carbono orgânico no solo. Resck (2005) relatam que solos com textura grosseira no Brasil apresentaram maior perda de COS quando cultivados, do que em outros solos de textura mais fina. Diante desta afirmativa, e tendo em vista a textura mais grosseira do solo deste estudo, torna-se cada vez mais importante estudar os sistemas agrícolas que apresentam texturas mais grosseiras, para identificaras formas de manejos que apresentam potencial para sequestrar carbono orgânico do solo, e assim ser considerada uma técnica abiótica (Lal, 2008). 
Tabela 3. Valores médios para argila $(<0,002 \mathrm{~mm})$, silte $(0,053-0,002 \mathrm{~mm})$, areia total $(2,00-0,053 \mathrm{~mm})$, areia grossa $(2,00$ -

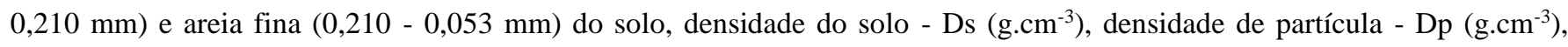
volume total de poros - VTP $(\%)$ do solo e temperatura do solo $\left({ }^{\circ} \mathrm{C}\right)$.

\begin{tabular}{lccc}
\hline & Argila & Silte & Areia total \\
\hline Mata & $302 \mathrm{~ns}$ & $148 \mathrm{~ns}$ & $547 \mathrm{~ns}$ \\
Linha de eucalipto & $315 \mathrm{~ns}$ & $116 \mathrm{~ns}$ & $563 \mathrm{~ns}$ \\
Bosque & $313 \mathrm{~ns}$ & $119 \mathrm{~ns}$ & $584 \mathrm{~ns}$ \\
\hline & Areia grossa & Areia fina & Temperatura \\
\hline Mata & $255 \mathrm{~ns}$ & $292 \mathrm{~ns}$ & $25 \mathrm{~b}$ \\
Linha de eucalipto & $253 \mathrm{~ns}$ & $317 \mathrm{~ns}$ & $28 \mathrm{a}$ \\
Bosque & $296 \mathrm{~ns}$ & $298 \mathrm{~ns}$ & $24 \mathrm{~b}$ \\
\hline & Ds & Dp & VTP \\
\hline Mata & $1,11 \mathrm{a}$ & $2,39 \mathrm{a}$ & $53,3 \mathrm{a}$ \\
Linha de eucalipto & $1,22 \mathrm{~b}$ & $2,44 \mathrm{a}$ & $49,7 \mathrm{~b}$ \\
Bosque & $1,25 \mathrm{~b}$ & $2,39 \mathrm{a}$ & $47,8 \mathrm{~b}$ \\
\hline
\end{tabular}

Obs.: Médias seguidas da mesma letra minúscula entre tratamentos na coluna não diferem entre si, pelo teste de Tukey-Kramer $(\mathrm{P}<0,05)$.

Fonte: Autores.

A temperatura apresentou diferença significativa entre os tratamentos (Tabela 3). A área de eucalipto do sistema ILP apresentou valor médio $\left(28^{\circ} \mathrm{C}\right)$ superior aos demais tratamentos. Tal resultado pode ser explica pelas copas rasas da área de eucalipto que permite a entrada de radiação solar. A temperatura do solo é de grande relevância para o funcionamento do ecossistema e a cobertura vegetal apresenta uma relação direta com a quantidade de radiação que atinge a superfície e a cobertura é definida pelo manejo da cultura (Veiga et al., 2010), os quais irão interferir no armazenamento da energia no solo (Carneiro et al., 2014).

Não houve diferença significativa na densidade do solo entre as posições de amostragem no sistema ILP entre o bosque e a linha de eucalipto. A densidade do solo apresentou diferença estatística para a mata nativa comparada aos demais tratamentos (Tabela 3). O resultado encontrado é o esperado, uma vez que, a mata primária é um ambiente sem modificações realizadas pelo homem, e permitem visualizar as modificações promovidas pela modificação no uso da terra. A conversão de mata nativa para agricultura pode provocar forte modificações na densidade do solo bem com gera a modificação na matéria orgânica do solo e o mecanismo-chave para aceleração deste processo é a erosão, que está sempre associado ao aumento na densidade do solo, que pode levar à aceleração a decomposição da matéria orgânica durante o transporte e deposição (Van Oost et al., 2007).

A densidade de partícula não apresentou diferença estatística $(\mathrm{P}<0,05)$ entre os tratamentos (Tabela 3). Os valores encontrados para a densidade de partícula podem ser conferidos a presença de 5,95\% $\mathrm{Fe}_{2} \mathrm{O}_{3}$ (Pires et al.,2012) que possui alta densidade de partículas, e a $11,83 \% \mathrm{Al}_{2} \mathrm{O}$ e $8,36 \% \mathrm{SiO}_{2}$ (Pires et al., 2012) que permite a estabilidade deste solo.

O volume total de poros apresentou valor superior para a mata nativa $(53,3 \%)$ comparando com os demais tratamentos, apresentando o mesmo comportamento da densidade do solo, o que é esperado, por estarem relacionados. A porosidade é necessária para o solo, e os valores encontrados estão entre 30 e $60 \%$, que indica que o uso da terra para a agricultura não promoveu a degradação da porosidade do solo, e não afeta a infiltração, retenção de água e trocas gasosas do solo. Este resultado é relevante, uma vez que de modo geral, a agricultura tem promovido, redução significativa na porosidade do solo (Anjos et. al., 1994; Silva; Mielniczuk, 1998). 


\subsection{Efeitos do sistema ILF nos teores e estoque de carbono orgânico do solo.}

A tabela 4 apresenta os resultados de análise de variância para o estoque e teores de carbono orgânico total do solo. Os resultados sinalizam que existem diferenças significativas entre os tratamentos. O carbono orgânico do solo e o estoque de carbono apresentam o mesmo comportamento. A mata nativa apresentou resultado superior aos demais tratamentos. Este resultado é espero, uma vez que a modificação, ao utilizar a terra gera a redução nos teores de carbono orgânico do solo. A conversão de mata primária para agricultura gera modificações ou perda nos teores e estoque de COS (Van Oost et al., 2007).

Tabela 4. Valores médios para Carbono orgânico do solo $\left(\mathrm{g} . \mathrm{kg}^{-1}\right)$ e estoque de carbono orgânico do solo $\left(\mathrm{g} . \mathrm{kg}^{-1}\right)$, em profundidade de $0,05 \mathrm{~m}$.

\begin{tabular}{lcc}
\hline Tratamentos & Carbono orgânico total do solo & Estoque de COS \\
\hline Mata primária & $15,0 \mathrm{a}$ & $19,9 \mathrm{a}$ \\
Linha de eucalipto & $12,3 \mathrm{~b}$ & $15,3 \mathrm{~b}$ \\
Bosque & $13,0 \mathrm{~b}$ & $15,8 \mathrm{~b}$ \\
\hline
\end{tabular}

Obs.: Médias seguidas da mesma letra minúscula entre tratamentos na coluna não diferem entre si, pelo teste de Tukey-Kramer $(\mathrm{P}<0,05)$. Fonte: Autores.

A ausência de diferença significativa entre os tratamentos sistema ILF e mata primaria para o estoque de COS (Figura 2), este resultado pode ser atribuído à ausência de revolvimento anual do solo, que proporcionou para a redução na decomposição de resíduos e conservação dos níveis de COS similares ao da mata primária. Estes resultados sugerem que o uso da terra para com sistemas ILP apresenta resiliência quanto ao armazenamento de COS, agindo como um sumidouro de $\mathrm{CO}_{2}$ atmosférico (Soto-Pinto et al., 2010).

Figura 2. Estoque de carbono orgânico do solo ( $\mathrm{t} / \mathrm{ha})$. Obs.: Médias seguidas da mesma letra maiúscula entre tratamentos não diferem entre si, pelo teste de Tukey-Kramer $(\mathrm{P}<0,05)$.

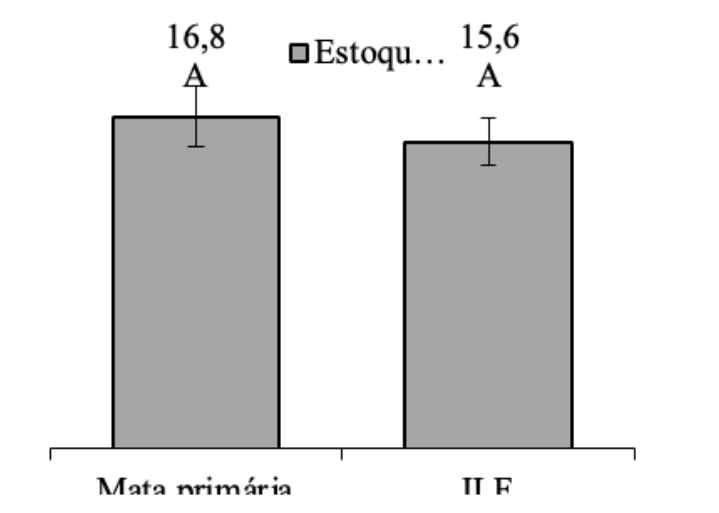

Fonte: Autores.

\section{Conclusão}

Os atributos físicos alterados pelo sistema ILP foram densidade do solo, volume total do solo e temperatura. A mata primaria apresentou teores e estoque de COS superior aos demais tratamentos. O estoque total de COS não apresentou diferença entre o sistema ILP e a mata primária. A partir destes resultados sugere a continuidade dos estudos comparando maiores profundidades tem em vista que os sistemas são arbóreos com sistema radicular profundo. 


\section{Referências}

Anjos, J. T.; Uberti, A. A. A.; Vizzotto, V. J.; Leite G. B. \& Krieger, M. (1994). Propriedades físicas em solos sob diferentes sistemas de uso e manejo. Revista Brasileira de Ciência do Solo, 18 (1), 139-145.

Assunção, S. A.; Pereira, M. G.; Rosset, J. S.; Berbara, R. L. L \& García, A. C. (2019) Carbon input and the structural quality of soil organic matter as a function of agricultural management in a tropical climate region of Brazil. Science of the Total Environment, 658 (1), 901-911.

Alvares, C. A., J. L. M.; Gonçalves, S. R.; Vieira, C.R. Silva \& W. Franciscatte, (2011). Spatial variability of physical and chemica lattributes of some forest soils in southe a stern of Brazil. Sci. Agric, 68, 697-705.

Balbino, L. C.; Barcellos, A. O. \& Stone, F. L. (2011) Marco referencial: integração lavoura-pecuária floresta (ILPf). Brasília, DF: Embrapa, 130.

Bayer, C.; Mielniczuk, J.; Amado, T. J. C.; Martinneto, L.; \& Fernandes; S. V. (2000) Organic matter storage in a Sandy clayloam Acrisol affected by tillage and cropping systems in southern Brazil. Soil Tillage Res, 54, 101-109.

Blake, G.R. \& Hartge, K. H. Bulk density. In: KLute A (Ed.). (1986) Methods of soil analysis: physical and miner alogical methods. Madison: ASA, 363375 .

Ballantyne A.P.; Smith. W.K.; Anderegg; W. R. L.; Kauppi, P.; Sarmiento, J.; Tans, P. P., Shevliakova; E., Pan, Y. \& Poulter, B. (2017) Accelerating net terrestrial carbon uptake during warming hiatus due to reduced respiration. Nature Climate Change, 7, $148-152$.

Bossuyt, H.; Six, J. \& Hendrix, P. F., (2005) Protection of soil carbon by microaggregates within earthworm casts. Soil Biol. Biochem, (2) 37, 251-258.

Camargo, O. A.; Moniz, A. C.; Jorge, J. A. \& Valadares, J. M. A. S. Métodos de análise química, mineralógica e física de solo do Instituto Agronômico de Campinas. Campinas, Instituto Agronômico, 1986. 94 p. (Boletim técnico, 106)

Chevallier, T.; Blanchart, E.; Albrecht, A. \& Feller, C. (2004) The physical protection of soil organic carbon in agrregates: a mechanism of carbon storage in a Vertisol under pasture and market gardening (Martinique, West Indies). Agric. Ecosyst. Environ. 103, 375-387.

Ferreira, C. D.; Moreira, F. T. de A.; Souto, P. C.; Alencar, L. dos S.; Borges, C. H. A. Soil organic carbon in a toposequence in the semiarid of Paraíba, Brazil. Research, Society and Development, 9 (5), p. e164953365, 2020.

Troian, D.; Rosset, J. S.; Ozório, J. M. B.; Castilho, S. C. P.; Marra, L. M. (2020) Carbono orgânico e estoque de carbono do solo em diferentes sistemas de manejo. Revista em Agronegócio e meio ambiente. 13 (2), 1447-1469.

Empresa Brasileira de Pesquisa Agropecuária. Centro Nacional de Pesquisa de Solos. (2006) Sistema brasileiro de classificação de solos. 2. ed. Rio de Janeiro, 306.

FAO, Global soil organic carbon map. http://www.fao.org/3/a-i8195e.pdf.

Hombegowda, H. C.; Van Straaten, O.; Köhler \& M., Hölscher, (2016) D. On the rebound: soil organic carbon stocks can bounce back to near forest levels when agroforests replace agriculture in southern India. Soil.

Ferreira, D. F. (2011) Sisvar 5.0: sistema de análises estatísticas. Lavras: UFLA.

INMET - Instituto Nacional de Meterologia, (2019). http://www.inmet.gov.br/portal/.

Lal, R. Soil science and the carbon civilization. (2007) Soil SCi. Soc. Am. J. 71 (5), 1425-1437.

Lal, R. Soil carbono sequestration to mitigate climate change. (2004) Encyclopedia of Energy, 123 (1-2), 289-298.

Lal, R. Carbon sequestration. Philosophical Transactions of the Royal Society, (2008). 363(1492), 815-830.

Piao, S.; Huang, M.; Liu; Z.; Wang, X.; Ciais, P.; Canadell, J.G.; Wang, K.; Bastos, A.; Friedlingstein, P. \& Houghton, R. A. (2018) Lower land-use emissions responsible for increased net land carbon sink during the slow warming period. Nature Geoscience, 11, 739-743.

Resck, D. V. S. (2005) O potencial de seqüestro de carbono em sistemas de produção de grãos sob plantio direto no Cerrado. In: Simpósio sobre Plantio Direto e Meio Ambiente: Seqüestro de Carbono e Qualidade da Água, 1. Foz do Iguaçu. Anais. Foz do Iguaçu: FEBRAPDP/Itaipu Nacional. 72-80.

Soto-Pinto, L. (2010) et al. Carbon sequestration through agroforestry in indigenous communities of Chiapas, Mexico. Agroforestry Systems, Dorchecht, 78 (1), 39-51.

Silva, I. F. \& Mielniczuk, J. (1998) Ação do sistema radicular de planta na formação e estabilização de agregados do solo. Revista Brasileira de Ciência do Solo, 21 (1), 113-117.

Vomocil, J. A. (1965) Porosity. In: BLACK, C. A. (Ed.). Methods of soil analysis: physical and mineralogical properties, including statistics of measurement and sampling. Madison: American Society of Agronomy, 499-510.

Van Oost, K. et al. (2007) The impact of agricultural soil erosion on the global carbon cycle. The American Association for the Advancement of Science, 318 (5850), 626-629.

Veiga, M.; Reinert \& D. J.; Reichert, J. M. (2010) Tillage systems and nutrient sources affecting soil cover, temperature and moisture in clayey Oxisol under corn. Revista Brasileira de Ciência do Solo, 34, 2011-2020.

Weimann C.; Farias, J.A.; Deponti G. \& deponti, G. (2017) Viabilidade econômica do componente arbóreo do sistema agrossilvipastoril comparada ao plantio floral na pequena propriedade rural. Pesquisa Florestal Brasileira, 37, 429-36. 